their degree work. In a lively discussion which followed, there was support for the idea of a National Certificate, and the difficulties in training isolated technicians, including those in schools, were stressed.

The final session dealt with postgraduate training. Summarizing the replies to the Institute's questionnaire, Dr. Wood said that few departments give any special courses in research methods, and that neither in botany nor zoology is it common for there to be concentration of the main resources of a department on a narrow field of inquiry. Dr. G. E. Fogg (University College, London) described a postgraduate course in microbiology, and Dr. G. Brownlee (King's College, London) a similar course in pharmacology. Both these consist of lectures and practical work. Prof. V. B. Wigglesworth (University of Cambridge) spoke on the Ph.D. and gave' as his opinion that, in spite of its obvious disadvantages, this degree is on the whole worth while; but that employers should not insist on it. $H_{\theta}$ deprecated the publication of theses, and Sir James Gray in the discussion went further and recommended their abolition. Dr. J. Y. Bogue (Imperial Chemical Industries, Ltd.) included, in his summary of the qualities which are desirable in the industrial research worker, a first-class education in the broadest sense, an ability to handle technicians and an ability to write English. The importance of the last was stressed by a number of speakers in the discussion, and other points that were made were that there might be scope for parttime postgraduate courses, and that workers who do not live in university towns are greatly handicapped by lack of library facilities. W. B. YAPP

\section{MOLECULAR REARRANGEMENTS IN ORGANIC CHEMISTRY}

$\mathrm{O}^{-1}$

April 6 a symposium was held by the Chemistry Department of Queen Mary College, London, on "Molecular Rearrangements in Organic Chemistry". Prof. D. H. Hey took the chair. The seven contributions covered those features of molecular rearrangements which ase of current interest and stimulated a lively discussion.

Prof. S. Winstein described his investigations of $1: 3$ and $1: 2$ hydrogen shifts in carbonium ions. Roberts and $\mathrm{Lee}^{1}$ had established the existence of a 1 : 3 hydrogen shift in the non-classical norbornyl carbonium ion; Prof. Winstein has found that the hydrogen shift does not accompany formation of the ion but occurs in a subsequent step. The first ion to be formed is the non-classical structure (I), and the subsequent migration involves as intermediate either the nortricyclonium ion (II), or the alternative structure (III). Prof. Winstein favoured the latter alternative. $\mathrm{He}$ then described experiments in the cyclohexane series which demonstrate the possibility of anchimeric assistance of ionization by adjacent hydrogen atoms, the ionization of appropriate cyclohexyl esters occurring more rapidly than would be anticipated and with a $1: 2$ shift of hydrogen.

In the subsequent discussion, Dr. H. Cardwell suggested that anchimeric assistance by adjacent hydrogen might be general in $S_{N} l$ solvolyses and pointed out that, if this were so, a statistical correction should be applied to observed solvolysis-rates, depending

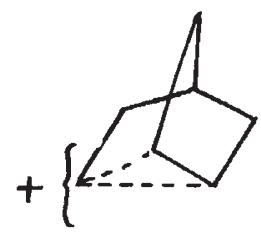

(I)

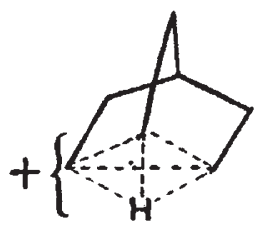

(II)

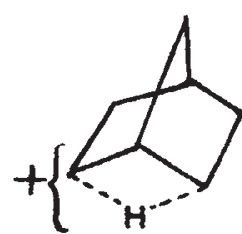

(III) on the number of hydrogen atoms able to participate. This idea was criticized by Prof. Winstein on experimental grounds. Prof. H. C. LonguetHiggins gave theoretical reasons in support of the structure (III) for the norbornyl intermediate. Prof. M. J. S. Dewar emphasized that, although anchimeric assistance of solvolysis implies greater stability of the $\pi$-complex form of a carbonium compound with the classical form, the converse is not necessarily true. After further discussion, the consensus of opinion seemed to be that anchimeric assistance of solvolysis by hydrogen does not occur, except in special cases, but that the $\pi$-complex ions with hydrogen in the non-classical position are probably more stable than isomeric classical structures.

Prof. C. W. Shoppee discussed the stereochemistry of Wagner rearrangements in terms of the mechanism suggested by Ingold and Shoppe ${ }^{2}$. In the rearrangement (IV), inversion should occur at both carbon atoms $\alpha, \beta$, and retention of configuration in the migrating group $R$. Prof. Shoppee described a number of rearrangements in the sterol series, involving migrations of groups or ring contraction, where the products obtained depend on the stereochemical configuration of the starting materials and can be predicted on the basis of the theory.

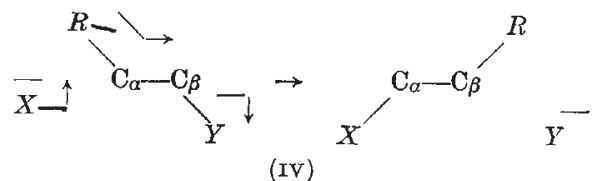

Prof. Winstein pointed out that these conclusions are somewhat too rigid; while the configuration of $R$ is invariably retained, and while $\mathrm{C}_{\alpha}$ and $\mathrm{C}_{\beta}$ commonly undergo inversion, cases are known where retention of configuration can occur. He pointed out that the stereochemical course of the reaction depends on the timing of the individual processes; in particular, exclusive inversion at $\mathrm{C}_{\beta}$ occurs as a rule only when there is anchimeric assistance of the ionization of $X$.

Dr. W. J. Hickinbottom then described a number of investigations into the course of rearrangements of epoxides and related glycols. These were interpreted in terms of the current carbonium ion mechanism. Interesting new reactions are the formation of allylic alcohols from epoxides in the presence of acid, and the fission of highly substituted epoxides and 1:2-glycols into lower ketones and olefins. These reactions were interpreted as follows :<smiles>CC(C)=C(C)C(C)(C)O</smiles>

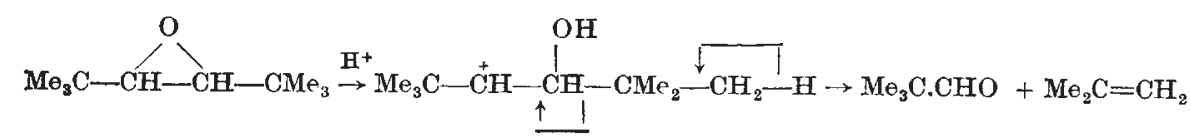


Whether or not the rearrangements of epoxides take place via intermediate glycols is being investigated.

The second session was devoted to anionotropy. Prof. W. G. Young described the reactions of allyl alcohol derivatives with thionyl chloride. Under conditions where intervention of chloride ion is avoided, the reactions proceed with rearrangement via cyclic transition states $\left(S_{N} i^{\prime}\right.$ mechanism), for example :

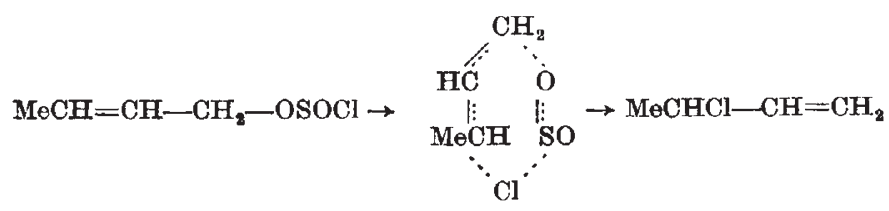

emphasized the difficulty of determining whether or not they are intramolecular. If they are, classical mechanisms will not suffice, for the rearrangements often occur between widely separated centres. $\mathrm{He}$ outlined the $\pi$-complex mechanism for such processes and applied the idea to the rearrangements of $\mathrm{N}$-bromoacetanilide and of hydrazobenzenes. Fresh experimental evidence was cited which suggested that the former reaction is intramolecular; and a general survey of the available evidence was held to support the mechanism in the case of hydrazobenzene derivatives. In the subsequent discussion, his conclusions concerning the mechanism of the N-bromoacetanilide rearrangement were strongly criticized and strongly defended.

It was generally agreed that the sym-

In the presence of chloride ion, an alternative $S_{N} 2$ reaction leads to unrearranged chloride. By suitable choice of the reaction conditions, either unrearranged chloride, or rearranged chloride, can be obtained. An $S_{N}$ l reaction can also be observed in ionizing solvents, the product from either isomeric alcohol then being an identical mixture of the two chlorides.

Dr. E. A. Braude reviewed the historical development of the concept of anionotropy, and emphasized the role of acid catalysis. He then described a reinvestigation of the rearrangement of $\alpha$-phenylallyl $p$-nitrobenzoate into cinnamyl $p$-nitrobenzoate in the presence of isotopically labelled $p$-nitrobenzoic acid in chlorobenzene solution. It was shown that the reaction occurs by two distinct paths, one involving an internal rearrangement (cf. (IV)) and one a bimolecular reaction with the added acid. Both reactions are catalysed by acid. Dr. Braude concluded that no free allylic cations are involved as intermediates, the bimolecular process being of the $S_{N} 2^{\prime}$ type ; and that free allyl cations are probably not involved in anionotropy (see also Nature, May 8, p. 863).

Prof. S. Winstein criticized Dr. Braude's conclusions on the grounds that free ions cannot in any event occur in the solvent used, and that the work described is consistent with the initial formation of an ion-pair, which can either rearrange by 'internal collapse' or react with $p$-nitrobenzoic acid. He thought that free ions might well participate in more polar solvents. A lively discussion followed, on the distinction between ion-pair mechanisms and mechanisms involving cyclic transition-states. The conclusion appeared to be that ion pairs and covalently bound cyclic intermediates are not qualitatively distinct, but form extremes in a graded series (cf. the relation between covalent and ionic bonds). In this case the experimental evidence does not lead to a clear distinction.

The final session dealt with rearrangements in the aromatic series. Prof. E. D. Hughes outlined a general classification of reactions in which groups migrate from the side-chain to the nucleus in an aromatic system. He concluded that the main types of mechanism are an intermolecular process of the $S_{N} 1$ or $S_{N} 2^{\prime}$ type, and an intramolecular process, giving $o$-substituted aniline derivatives via cyclic transition states. The rearrangement of N-phenylhydroxylamine was cited as an example of the former, and the rearrangement of $\mathrm{N}$-nitranilines, and the reaction of aniline with sulphur trioxide, as examples of the latter.

Prof. M. J. S. Dewar outlined the various types of rearrangements that occur in aromatic systems, and

posium had been of value both in presenting various points of view and in clarifying ideas. A remarkable measure of agreement was reached on most of the controversial issues.
M. F. Anselul

M. J. S. DEwar

1 Roberts, J. D., and Lee, C. C., J. Amer. Chem. Soc., 73, 5009 (1951). ${ }^{2}$ Ingold, C. K., and Shoppee, C. W., J. Chem. Soc., 365 (1928).

\section{FRICTION IN RELATION TO RHEOLOGICAL PROPERTIES}

LTHOUGH a number of theories of solid friction A have in the past been proposed, the one which has been most fruitful and most widely accepted in recent years is the adhesion mechanism of friction originally developed for sliding metals. According to this model, metals, when placed in contact, touch only at their high spots, and the local pressures are usually sufficient to produce plastic flow. At these regions strong adhesion occurs by a process which may be called cold welding. The strong junctions thus formed must be sheared if sliding is to occur, and this is primarily the source of the frictional resistance. With metals the deformation of the asperities is primarily plastic. Under these conditions the area of contact is proportional to the load and independent of the size of the bodies, so that the friction, too, is proportional to the load and independent of the size of the bodies (Amontons's laws).

The reality of junction formation is shown by examining the friction tracks, which provide clear evidence of welding and shearing. This raises the interesting question as to why normal adhesion is not observed between metals. Although contaminant films may be partly responsible, the main effect appears to be due to released elastic stresses which peel the surfaces apart as the joining load is removed, so that little or no normal adhesion is detected. With soft ductile materials such as indium, where the metal can take up changes in shape of the surfaces as the load is removed, large adhesions are observed.

\section{Clean Metals and the Influence of Oxide Films}

If metal surfaces are thoroughly denuded of oxide films, Amontons's laws no longer hold. Because the surfaces are clean, they adhere strongly wherever they touch and the sliding process itself produces a very marked growth of junctions. Under favourable conditions, junction growth may proceed until the 